\title{
Sign-changing solutions for some nonlinear problems with strong resonance
}

\author{
Aixia Qian
}

Correspondence: qaixia@amss.ac.cn School of Mathematic Sciences, Qufu Normal University, Qufu Shandong, 273165, P. R. of China

\section{Abstract}

By means of critical point and index theories, we obtain the existence and multiplicity of sign-changing solutions for some elliptic problems with strong resonance at infinity, under weaker conditions.

2000 Mathematics Subject Classification: 35J65; 58 E05.

Keywords: critical point theory, strong resonance, index theory, Cerami condition

\section{Introduction}

In this article, we consider the following equation,

$$
\left\{\begin{array}{c}
-\Delta u=f(u), \\
u \in H_{0}^{1}(\Omega) .
\end{array}\right.
$$

where $\Omega$ is a bounded domain in $\mathbb{R}^{n}$ with smooth boundary $\partial \Omega$. In order to explain what we mean, a brief description is necessary. We suppose that $f$ is asymptotically linear, i.e., $\lim _{|u| \rightarrow \infty} \frac{f(u)}{u}$ exists. If we set

$$
\alpha:=\lim _{|u| \rightarrow \infty} \frac{f(u)}{u},
$$

then we can write

$$
f(u)=\alpha u-g(u)
$$

with

$$
\frac{g(u)}{u} \rightarrow 0 \text { as }|u| \rightarrow \infty
$$

We denote $\lambda_{1}<\lambda_{2}<\ldots<\lambda_{j}<\ldots$ to be the distinct eigenvalues sequence of $-\Delta$ with the Dirichlet boundary conditions. We state that problem (1.1) is resonant at infinity if $\alpha$ in (1.2) is an eigenvalue $\lambda_{k}$. The situation

$$
\lim _{|u| \rightarrow \infty} g(u)=0 \text { and } \lim _{|u| \rightarrow \infty} \int_{0}^{u} g(t) d t=\beta \in \mathbb{R}
$$

is what we call a strong resonance. 
Now we present some of the results of this article. We write (1.1) in the following form:

$$
\left\{\begin{array}{l}
-\Delta u-\lambda_{k} u+g(u)=0 \\
u \in H_{0}^{1}(\Omega)
\end{array}\right.
$$

We assume that $g$ is a smooth function satisfying the following conditions.

$\left(g_{1}\right) g(t) \cdot t \rightarrow 0$ as $|t| \rightarrow \infty$.

$\left(g_{2}\right)$ the real function $G(t)=\int_{0}^{t} g(s) d s$ is well defined and $G(t) \rightarrow 0$ as $t \rightarrow+\infty$.

$\left(g_{3}\right) G(t) \geq 0, \forall t \in \mathbb{R}$.

Theorem 1.1 If $\left(g_{1}\right)-\left(g_{3}\right)$ hold, then problem (1.1) has at least one solution.

Remark 1.1 Since 0 is a particular point, we cannot make sure those solutions are nontrivial without more conditions.

Theorem 1.2 Let $g(0)=0$, and suppose that $\left(g_{1}\right)-\left(g_{3}\right)$ hold, and

$$
g^{\prime}(0)=\sup \left\{g^{\prime}(t): t \in \mathbb{R}\right\}
$$

then problem (1.3) has at least one sign-changing solution.

Theorem 1.3 Assume that $\left(g_{1}\right)\left(g_{3}\right)$ hold, $g$ is odd, and $G(0) \geq 0$. Moreover, suppose that there exists an eigenvalue $\lambda_{h}<\lambda_{k}$ s.t.

$$
g^{\prime}(0)+\lambda_{h}-\lambda_{k}>0
$$

Then, problem (1.3) possess at least $m=\operatorname{dim}\left(M_{h} \oplus \ldots \oplus M_{k}\right)-1$ distinct pairs of sign-changing solutions $\left(M_{j}\right.$ denotes the eigenspace corresponding to $\left.\lambda_{j}\right)$.

Remark 1.2 In the article [1], they only show the existence of solutions to problem (1.3), while we obtain its sign-changing solutions under the same conditions.

The resonance problem has been widely studied by many authors using various methods-see [1-6] and the references therein. We will use critical point and pseudoindex theories to obtain the sign-changing solutions for strong resonant problem (1.3). We also allow the case in which resonance also occurs at zero.

In Section 2, we will give some preliminaries, which are fundamental for this article. In Section 3, we will give some abstract critical point theorems, which are used to prove above theorems in this article. In Section 3, we prove our main theorems, which result in the existence and multiplicity of sign-changing solutions.

\section{Preliminaries}

We denote by $X$ a real Banach space. $B_{R}$ denotes the closed ball in $X$ centered at the origin and with radius $R>0$. $J$ is a continuously Frèchet differentiable map from $X$ to $\mathbb{R}$, i.e., $J \in C^{1}(X, \mathbb{R})$.

In the literature, deformation theorems have been proved under the assumption that $J \in C^{1}(X, \mathbb{R})$ satisfies the well-known Palais-Smale condition. In problems which do not have resonance at infinity, the (PS) condition is easy to verify. On the other hand, a weaker condition than the condition (PS) is needed to study problems with strong resonance at infinity.

Definition 2.1 We state that $J \in C^{1}(X, \mathbb{R})$ satisfies the condition $(C)$ in $] c_{1}, c_{2}[(-\infty \leq$ $\left.c_{1}<c_{2} \leq+\infty\right)$ if

(i) every bounded sequence $\left\{u_{k}\right\} \subset J^{1}(] c_{1}, c_{2}[)$, for which $\left\{J\left(u_{k}\right)\right\}$ is bounded and $J\left(u_{k}\right)$ $\rightarrow 0$, possesses a convergent subsequence, and 
(ii) $\forall c \in] c_{1}, c_{2}[, \exists \sigma, R, \alpha>0$ s.t. $[c-\sigma, c+\sigma] \subset] c_{1}, c_{2}\left[\right.$ and $\forall u \in J^{1}([c-\sigma, c+\sigma])$, $\|u\| \geq R:\|\gamma(u)\|\|u\| \geq \alpha$.

In the article [1], they propose a deformation theorem under the condition (C). For $c$ $\in \mathbb{R}$, denote

$$
A_{c}=\{u \in X: J(u) \leq c\}, \quad K_{c}=\left\{u \in X: J^{\prime}(u)=0, J(u)=c\right\} .
$$

Proposition 2.2 [1] Let $X$ be a real Banach space, and let $J \in C^{1}(X, \mathbb{R})$ satisfy the condition $(C)$ in $] c_{1}, c_{2}$ [. If $\left.c \in\right] c_{1}, c_{2}$ [ and $N$ is any neighborhood of $K_{c}$, then there exists a bounded homeomorphism $\eta$ of $X$ onto $X$ and constants $\bar{\varepsilon}>\varepsilon>0$, s.t. $[c-\bar{\varepsilon}, c+\bar{\varepsilon}] \subset] c_{1}, c_{2}[$ satisfying the following properties:

(i) $\eta\left(A_{c+\varepsilon} \mid N\right) \subset A_{c-\varepsilon}$.

(ii) $\eta\left(A_{c+\varepsilon}\right) \subset A_{c-\varepsilon}$, if $K_{c}=\varnothing$.

(iii) $\eta(x)=x$, if $x \notin J^{-1}([c-\bar{\varepsilon}, c+\bar{\varepsilon}])$.

Moreover, Let $G$ be a compact group of (linear) unitary transformation on a real Hilbert space $H$. Then,

(vi) $\eta$ can be chosen to be G-equivariant, if the functional $J$ is $G$-invariant. Particularly, $\eta$ is odd if the functional $J$ is even.

\section{Abstract critical point theorems}

In this article, we shall obtain solutions of problem (1.3) using the linking-type theorem. Its different definitions can be seen in $[1,7,8]$ and the references therein.

Definition 3.1 Let $H$ be a real Hilbert space and $A$ a closed set in $H$. Let $B$ be an Hilbert manifold with boundary $\partial B$, we state that $A$ and $\partial B$ link if

(i) $A \cap \partial B=\varnothing$;

(ii) If $\varphi$ is a continuous map of $H$ into itself s.t. $\varphi(u)=u, \forall u \in \partial B$, then $\varphi(B) \cap A \neq$ $\varnothing$.

There are some typical examples as following, cf. [1,7,9].

Example 3.1 Let $H_{1}$ and $H_{2}$ be two closed subspaces of $H$ such that

$$
H=H_{1} \oplus H_{2}, \quad \operatorname{dim} H_{2}<\infty .
$$

Hence, if $A=H_{1}, B=B_{R} \cap H_{2}$, then, $A$ and $\partial B$ link.

Example 3.2 Let $H_{1}$ and $H_{2}$ be two closed subspaces of $H$ such that $H=H_{1} \oplus H_{2}$, $\operatorname{dim} H_{2}<\infty$, and consider $e \in H_{1},\|e\|=1,0<\rho<R_{1}, R_{2}$, set

$$
A=H_{1} \cap S_{\rho}, \quad B=\left\{u=v+t e: v \in H_{2} \cap B_{R_{2}}, 0 \leq t \leq R_{1}\right\} .
$$

Then, $A$ and $\partial B$ link.

Let $X \subset H$ be a Banach space densely embedded in $H$. Assume that $H$ has a closed convex cone $P_{H}$ and that $P:=P_{H} \cap X$ has interior points in $X$. Let $J \in C^{1}(H, \mathbb{R})$. In the article [10], those authors construct the pseudo-gradient flow $\sigma$ for $J$, and have the same definition as [11].

Definition 3.1 Let $W \subset X$ be an invariant set under $\sigma$. $W$ is said to be an admissible invariant set for $J$ if (a) $W$ is the closure of an open set in $X$; (b) if $u_{n}=\sigma\left(t_{n}, v\right) \rightarrow u$ in 
$H$ as $t_{n} \rightarrow \infty$ for some $v \notin W$ and $u \in K$, then $u_{n} \rightarrow u$ in $X$; (c) If $u_{n} \in K \cap W$ is such that $u_{n} \rightarrow u$ in $H$, then $u_{n} \rightarrow u$ in $X$; (d) For any $u \in \partial W \backslash K$, we have $\sigma(t, u) \in \stackrel{\circ}{W}$ for $t>0$.

Now let $S=X \backslash W, W=P \cup(-P)$. Similar to the proof described in the article [10], the $W$ is an admissible invariant set for $J$ in the following section 4 . We define

$$
\begin{aligned}
\phi^{*}=\{\Gamma \mid \Gamma(t, x):[0,1] \times X & \rightarrow X \text { is continuous in the } X \text { - topology and } \\
& \Gamma(t, W) \subset W\} .
\end{aligned}
$$

In the article [7], a new linking theorem is given under the condition (PS). Since the deformation still holds under the condition (C) (see [1]), the following theorem also holds.

Theorem 3.1 Suppose that $W$ is an admissible invariant set of $J$ and $J \in C^{1}(H, \mathbb{R})$ such that

$\left(J_{1}\right) J$ satisfies condition $(C)$ in $] 0,+\infty[$;

$\left(J_{2}\right)$ There exists a closed subset $A \subset H$ and a Hilbert manifold $B \subset H$ with boundary $\partial B$ satisfying

(a) there exist two constants $\beta>\alpha \geq 0$ s.t.

$$
J(u) \leq \alpha, \forall u \in \partial B ; \quad J(u) \geq \beta, \forall u \in A
$$

i.e., $a_{0}:=\sup _{\partial B} J \leq b_{0}:=\inf _{A} J$.

(b) $A$ and $\partial B$ link;

(c) $\sup _{u \in B} J(u)<+\infty$.

Then, $a^{*}$ defines below is a critical value of $J$

$$
a^{*}=\inf _{\Gamma \in \phi^{*}} \sup _{\Gamma([0,1], A) \cap S} J(u) .
$$

Furthermore, assume $0 \notin K_{a^{*}}$, then $K_{a^{*}} \cap S \neq \varnothing$, if $a^{*}>b_{0}$ and $K_{a^{*}} \cap A \neq \varnothing$, if $a^{*}=$ $b_{0}$.

In this article, we shall consider the symmetry given by a $\mathbb{Z}_{2}$ action, more precisely even functionals.

Theorem 3.2 Suppose $J \in C^{1}(H, \mathbb{R})$ and the positive cone $P$ is an admissible invariant for $J, K_{c} \cap \partial P=\varnothing$, for $c>0$, such that

$\left(J_{1}\right) J$ satisfies condition $(C)$ in $] 0,+\infty[$, and $J(0) \geq 0$;

$\left(J_{2}\right)$ There exist two closed subspace $H^{+}, H^{-}$of $H$, with codim $H^{+}<+\infty$ and two constants $c_{\infty}>c_{0}>J(0)$ satisfying

$$
J(u) \geq c_{0}, \forall u \in S_{\rho} \cap H^{+} ; \quad J(u)<c_{\infty}, \forall u \in H^{-} .
$$

$\left(J_{3}\right) J$ is even.

Hence, if $\operatorname{dim} H^{-}>\operatorname{codim} H^{+}+1$, then $J$ possesses at least $m:=\operatorname{dim} H^{-}$-codim $H^{+}-1$ 
( $m:=\operatorname{dim} H^{-}-1$ resp.) distinct pairs of critical points in $X \backslash P \cup(-P)$ with critical values belong to $\left[c_{0}, c_{\infty}\right]$.

Remark 3.1 The above theorem locates the critical points more precisely than Theorem 3.3 in [10].

We shall use pseudo-index theory to prove Theorem 3.2. First, we need the notation of genus and its properties, see [10,12]. Let

$$
\Sigma_{X}=\{A \subset X: A \text { is closed in } X, A=-A\} ;
$$

with more preciseness, we denote $i_{X}(A)$ to be the genus of $A$ in $X$.

Proposition 3.2 Assume that $A, B \in \Sigma_{X}, h \in C(X, X)$ is an odd homeomorphism, then

(i) $i_{X}(A)=0$ if and only if $A=\varnothing$;

(ii) $A \subset B \Rightarrow i_{X}(A) \leq i_{X}(B)$ (monotonicity);

(iii) $i_{X}(A \cup B) \leq i_{X}(A)+i_{X}(B)$ (subadditivity);

(iv) $i_{X}(A) \leq i_{X}(\overline{h(A)})$ (supervariancy);

(v) if $A$ is a compact set, then $i_{X}(A)<+\infty$ and there exists $\delta>0$ s.t. $i_{X}\left(N_{\delta}(A)\right)=i_{X}$

$(A)$, where $N_{\delta}(A)$ denotes the closed $\delta$ - neighborhood of $A$ (continuity);

(vi) if $i_{X}(A)>k, V$ is a $k$-dimensional subspace of $X$, then $A \cap V^{\perp} \neq \varnothing$;

(vii) if $W$ is a finite dimensional subspace of $X$, then $i_{X}\left(h\left(S_{\rho}\right) \cap W\right)=\operatorname{dim} W$.

(viii) Let $V, W$ be two closed subspaces of $X$ with codim $V<+\infty, \operatorname{dim} W<+\infty$.

Hence, if $h$ is bounded odd homeomorphism on $X$, then we have

$$
i_{X}\left(W \cap h\left(S_{\rho} \cap V\right)\right) \geq \operatorname{dim} W-\operatorname{codim} V .
$$

The proposition is still true when we replace $\Sigma_{X}$ by $\Sigma_{H}$ with obvious modification.

Proposition $3.3[10,11]$ If $A \in \Sigma_{X}$ with $2 \leq i_{X}(A)<\infty$, then $A \cap S \neq \varnothing$.

Proposition 3.4 Let $A \in \Sigma_{H}$, then $A \cap X \in \Sigma_{X}$ and $i_{H}(A) \geq i_{X}(A \cap X)$.

Now, we shall discuss about the notion of pseudo-index.

Definition 3.2 [1] Let $I=(\Sigma, \mathcal{H}, i)$ be an index theory on $H$ related to a group $G$, and $B \in \Sigma$. We call a pseudo-index theory (related to $B$ and $I$ ) a triplet

$$
I^{*}=\left(B, \mathcal{H}^{*}, i^{*}\right)
$$

where $\mathcal{H}^{*} \subset \mathcal{H}$ is a group of homeomorphism on $H$, and $i^{*}: \Sigma \rightarrow \mathbb{N} \cup\{+\infty\}$ is the map defined by

$$
i^{*}(A)=\min _{h \in \mathcal{H}^{*}} i(h(A) \cap B) .
$$

Proof of Theorem 3.2 Consider the genus $I=(\Sigma, \mathcal{H}, i)$ and the pseudo-index theory relate to $I$ and $B=S_{\rho} \cap H^{+}, I^{*}=\left(S_{\rho} \cap H^{+}, \mathcal{H}^{*}, i^{*}\right)$, where

$$
\begin{aligned}
& \mathcal{H}^{*}=\{h \mid h \text { is an odd }- \text { bounded homeomorphism on } H \text { and } h(u)=u \text { if } \\
& \left.\qquad u \notin J^{-1}(] 0,+\infty[)\right\} .
\end{aligned}
$$

Obviously, conditions $\left(a_{1}\right)\left(a_{2}\right)$ of Theorem 2.9 [1] are satisfied with $a=0, b=+\infty$ and $b=S_{\rho} \cap H^{+}$. Now, we prove the condition that $\left(a_{3}\right)$ is satisfied with $\bar{A}=H^{-}$. It is obvious that $\left.\left.\bar{A} \subset J^{-1}(]-\infty, c_{\infty}\right]\right)$, and by property (iv) of genus, we have 


$$
\begin{aligned}
i^{*}(\bar{A})=i^{*}\left(H^{-}\right) & =\min _{h \in \mathcal{H}^{*}} i\left(h\left(H^{-}\right) \cap S_{\rho} \cap H^{+}\right) \\
& =\min _{h \in \mathcal{H}^{*}} i\left(H^{-} \cap h^{-1}\left(S_{\rho} \cap H^{+}\right)\right) .
\end{aligned}
$$

Now, by (viii) of Proposition 3.2, we have

$$
i\left(H^{-} \cap h^{-1}\left(S_{\rho} \cap H^{+}\right)\right) \geq \operatorname{dim} H^{-}-\operatorname{codim} H^{+} .
$$

Therefore we get

$$
i^{*}(\bar{A}) \geq \operatorname{dim} H^{-}-\operatorname{codim} H^{+} .
$$

Then, by Theorem 2.9 in [11] and Proposition 3.3 above, the numbers

$$
c_{k}=\inf _{A \in \Sigma_{k}} \sup _{u \in A \cap S} J(u), \quad k=2, \ldots, \operatorname{dim} H^{-}-\operatorname{codim} H^{+} .
$$

are critical values of $J$ and

$$
J(0)<c_{0} \leq c_{k} \leq c_{\infty}, \quad k=2, \ldots, \operatorname{dim} H^{-}-\operatorname{codim} H^{+} .
$$

If for every $k, c_{k} \neq c_{k+1}$, then we get the conclusion of Theorem 3.2. Assume now that

$$
c=c_{k}=\cdots=c_{k+r} \text { with } r \geq 1 \text { and } k+r \leq \operatorname{dim} H^{-}-\operatorname{codim} H^{+} .
$$

Then, similar to the proof of Theorem 2.9 [11], where $K_{c}$ is replaced by $K_{c} \cap S$ and $A$ by $A \cap S$, we have

$$
i\left(K_{c} \cap S\right) \geq r+1 \geq 2
$$

Now, from Proposition 3.3 and (3.1), we deduce that

$$
0 \notin K_{c} \cap S .
$$

Since a finite set (not containing 0) has genus 1, we deduce from (3.2) and (3.3) that $K_{c}$ above contains infinitely many sign-changing critical points. Therefore, $J$ has at least $m:=\operatorname{dim} H^{-}$-codim $H^{+}-1$ distinct pairs of sign-changing critical points in $X \backslash P \cup(-P)$ with critical values belonging to $\left[c_{0}, c_{\infty}\right]$.

If codim $H^{+}=0$, then we consider $c_{j}$ for $j \geq 2$. As per the above arguments, $J(0)<c_{0} \leq c_{2} \leq c_{3} \leq \cdots \leq c_{\operatorname{dim} H^{-}} \leq c_{\infty}$ and if $c:=c_{j}=\ldots=c_{j+l}$ for $2 \leq j \leq j+l \leq$ $\operatorname{dim} H^{-}$with $l \geq 1$, then $i\left(K_{c} \cap S\right) \geq l+1 \geq 2$.

Therefore, $J$ has at least $\operatorname{dim} H-1$ pairs of sign-changing critical points with values belong to $\left[c_{0}, c_{\infty}\right]$.

Remark 3.2 Theorem 3.1 above can also be proved by the pseudo-index theory in the same way as Theorem 3.2.

\section{Proof of Theorems 1.1-1.3}

We shall apply the abstract results of Section 3 to problem (1.3). Let $H:=H_{0}^{1}(\Omega)$, $X:=C_{0}^{1}(\Omega)$. Clearly the solutions of problem (1.3) are the critical points of the functional 


$$
J(u)=\frac{1}{2}\left(\|u\|^{2}-\lambda_{k}|u|^{2}\right)+\int_{\Omega} G(u) d x,
$$

where $|\cdot|$ denotes the norm in $L^{2}(\Omega)$, and therefore, $J \in C^{1}(H, \mathbb{R})$. We denote by $M_{j}$ the eigenspace corresponding to the eigenvalue $\lambda_{j}$. If $m \geq 0$ is an integer number, set

$$
H^{-}(m)=\oplus_{j \leq m} M_{j}
$$

$H^{+}(m)=$ closure in $H_{0}^{1}(\Omega)$ of the linear space spanned by $\left\{M_{j}\right\}_{j \geq m}$.

Clearly $H^{+}(m) \cap H^{-}(m)=M_{m}$.

Proposition 4.1 [1] If $\left(g_{1}\right),\left(g_{2}\right)$ hold, then the functional $J$ defined by (4.1) satisfies the condition $(C)$ in $] 0,+\infty[$.

Proof of Theorem 1.1 If $G(0)=0$, then by $\left(g_{3}\right), G$ takes its minimum at 0 , so that $g$ $(0)=0$ and 0 is a solution of (1.3). We assume that $G(0)>0$. Similar to the proof as for the case in [1], there exists $R, \gamma>0$ such that

$$
\begin{gathered}
J(u) \geq \gamma, \quad u \in H^{+}(k+1) ; \\
J(u) \leq \frac{\gamma}{2}, \quad u \in H^{-}(k) \cap S_{R} .
\end{gathered}
$$

Let $\partial B=H^{-}(k) \cap S_{R}, A=H^{+}(k+1)$, then by Example 3.1 we get that $\partial B$ and $A$ link, and $J$ is bounded on $B=H^{-}(k) \cap B_{R}$. Moreover, by Proposition 4.1, $J$ satisfies condition (C) in ]0, $+\infty[$. Therefore, the conclusion of Theorem 1.1 follows by Theorem 3.1.

Remark 4.1 If $J(0)=0$, then the solutions obtained in Theorem 1.1 are sign-changing ones.

Proof of Theorem 1.2 Since $g(0)=0, u(x)=0$ is a solution of (1.3). In this case, we are interested in finding the existence of sign-changing solutions to problem (1.3). The case $g(t)=0, \forall t \in \mathbb{R}$ is trivial. We assume that $g(t) \neq 0$ for some $t$. Then, it is easy to see that $\left(g_{2}\right),\left(g_{3}\right)$ and (1.4) imply $g^{\prime}(0)>0$. Similar to the proof as for Theorem 5.1 [1], each of the following holds:

$$
\lambda_{1}-\lambda_{k}+g^{\prime}(0)>0
$$

where $\lambda_{k} \neq \lambda_{1}$ and there exists $\lambda_{h} \in \sigma(-\Delta)$ with $\lambda_{2} \leq \lambda_{h} \leq \lambda_{k}$ such that

$$
\lambda_{h}-\lambda_{k}+g^{\prime}(0)>0, \quad \frac{1}{2}\left(\lambda_{h-1}-\lambda_{k}\right) t^{2}+G(t) \leq G(0) \quad \forall t \in \mathbb{R} .
$$

Under (4.1), there exist three positive constants $\rho<R, \gamma$ such that

$$
\begin{gathered}
J(u) \geq J(0)+\gamma, \quad u \in S_{\rho} ; \\
J(e) \leq J(0)+\frac{\gamma}{2}, \quad e \in M_{1} \cap S_{\rho} .
\end{gathered}
$$

Since $J(0)=G(0) \cdot|\Omega| \geq 0(|\Omega|$ is the Lebesgue measure of $\Omega)$, we have

$$
0<J(0)+\frac{\gamma}{2}<J(0)+\gamma
$$

Fix $e \in M_{1} \cap S_{\rho}$, set

$$
A=S_{\rho} ; \quad B=\{t e: t \in[0, R]\} .
$$


Then, by Example 3.1, $A$ and $\partial B$ link and $J$ is bounded on $B$. Moreover, by Proposition 4.1, $J$ satisfies condition $(C)$ in $] 0,+\infty[$. Then, by Theorem 3.1, $J$ possesses a critical point $u_{0}$ such that $J\left(u_{0}\right) \geq J(0)+\gamma$. So $u_{0}$ is a sign-changing solution to problem (1.3).

Under (4.3) with similar arguments as given above, we get

$$
\begin{gathered}
J(u) \geq J(0)+\gamma, \quad u \in H^{+}(h) \cap S_{\rho} ; \\
J(u) \leq J(0)+\frac{\gamma}{2}, \quad u \in \partial B(h, R) .
\end{gathered}
$$

where $B(h, R)=\left\{u+t e: u \in H^{-}(h-1) \cap B_{R}, e \in M_{h} \cap S_{1}, 0 \leq t \leq R\right\}$. Set

$$
A=H^{+}(h) \cap S_{\rho}, \quad B=B(h, R) .
$$

Then, by Example 3.2, $A$ and $\partial B$ link and $J$ is bounded on $B$. Moreover, by Proposition 4.1, $J$ satisfies condition $(C)$. Using Theorem 3.1, we can conclude that $J$ possesses a sign-changing critical point $u_{0}$ with $J\left(u_{0}\right) \geq J(0)+\gamma$.

Remark 4.2 If $g^{\prime}(0)=0$, i.e., resonance at 0 is allowed, then by using an argument similar to that in the proof of Theorem 1.2, problem (1.3) still has at least a sign-changing solution under these conditions: Let $g(0)=0$. Assume that $\left(g_{1}\right),\left(g_{2}\right)$ hold and

$$
G(t)>0, \quad \forall t \neq 0, \quad G(0)=0 .
$$

Moreover, suppose that either of the following holds:

$$
\begin{gathered}
\lambda_{k}=\lambda_{1} ; \\
\lambda_{k} \neq \lambda_{1} \text { and } \frac{1}{2}\left(\lambda_{k-1}-\lambda_{k}\right) t^{2}+G(t) \leq 0 \quad \text { for } \forall t \in \mathbb{R} .
\end{gathered}
$$

Proof of Theorem 1.3 By Proposition 3.1 and Lemma 5.3 [1], the assumptions of Theorem 3.2 are satisfied with

$$
H^{+}=H^{+}(h), \quad H^{-}=H^{-}(k) .
$$

Thus, there exist at least

$$
\operatorname{dim} H^{-}-\operatorname{codim} H^{+}-1=\operatorname{dim}\left\{M_{h} \oplus \cdots M_{k}\right\}-1
$$

distinct pairs of sign-changing solutions of problem (1.3).

Remark 4.3 We also allow resonance at zero in problem (1.3). By using Theorem 3.2 and Lemma 5.4 [1], we have assumed that $g$ is odd and that $\left(g_{1}\right)\left(g_{2}\right)$ are satisfied. Suppose in addition

$$
G(t)>0 \text { for } \forall t \neq 0 \text { and } G(0)=0 .
$$

Then, the problem (1.3) possesses at least $\operatorname{dim} M_{k}-1$ distinct pairs of sign-changing solutions. $\left(M_{k}\right.$ denotes the eigenspace corresponding to $\lambda_{k}$ with $\left.k \geq 2\right)$

The author is grateful to the anonymous referee for his or her suggestions. This study was supported by the Chinese National Science Foundation $(11001151,10726003)$, the National Science Foundation of Shandong (Q2008A03) and the Science Foundation of China Postdoctoral (201000481301) and Shandong Postdoctoral. 


\section{References}

1. Bartolo, P, Benci, V, Fortunato, D: Abstract critical point theorems and applications to some nonlinear problems with strong resonance at infinity. Nonlinear Anal. 7, 981-1012 (1983). doi:10.1016/0362-546X(83)90115-3

2. Bartsch, $T, L i, S J$ : Critical point theory for asymptotically quadratic functionals and applications to problems with resonance. Nonlinear Anal. 28, 419-441 (1997). doi:10.1016/0362-546X(95)00167-T

3. Li, SJ, Liu, JQ: Computations of critical groups at degenerate ciritical point and applications to nonlinear differentia equations with resonace. Houson J Math. 25, 563-582 (1999)

4. Molle, R, Passaseo, D: Nonlinear elliptic equations with large supercritical exponents. Calc Var. 26, 201-225 (2006). doi:10.1007/s00526-005-0364-3

5. Qian, AX: Neumann problem of elliptic equation with strong resonance. Nonlinear Anal TMA. 66, 1885-1898 (2007). doi:10.1016/j.na.2006.02.046

6. Su, JB, Zhao, LG: An elliptic resonant problem with multiple solutions. J Math Anal Appl. 319, 604-616 (2006) doi:10.1016/j.jmaa.2005.10.059

7. Schechter, M, Wang, ZQ, Zou, WM: New linking theorem and sign-changing solutions. Commun Partial Equ. 29 471-488 (2004). doi:10.1081/PDE-120030405

8. Struwe, M: Variational Methods. Springer, 2 (1990)

9. Schechter, M: Resonance problems with respect to the Fučik spectrum. Trans Am Math Soc. 352, $4195-4205$ (2000). doi:10.1090/S0002-9947-00-02655-6

10. Qian, AX, Li, SJ: Multiple nodal soltuions for elliptic equations. Nonlinear Anal. 37, 615-632 (2004)

11. Li, SJ, Wang, ZQ: Ljusternik-Schnirelman theory in partially ordered Hilbert spaces. Trans Am Math Soc. 354, 3207-3227 (2002). doi:10.1090/S0002-9947-02-03031-3

12. Rabinowitz, P: Minimax methods in critical point theory with applications to differential equations. CBMS Reg Cof Ser Math. 65 (1986)

doi:10.1186/1687-2770-2011-18

Cite this article as: Qian: Sign-changing solutions for some nonlinear problems with strong resonance. Boundary Value Problems 2011 2011:18.

\section{Submit your manuscript to a SpringerOpen ${ }^{\circ}$} journal and benefit from:

Convenient online submission

- Rigorous peer review

- Immediate publication on acceptance

- Open access: articles freely available online

- High visibility within the field

- Retaining the copyright to your article

Submit your next manuscript at $>$ springeropen.com 